\title{
p63 Expression in Solitary and Syndromic Odontogenic Keratocysts: An Immunohistochemical Study ${ }^{\dagger}$
}

\author{
Elisa Luconi ${ }^{1, *}$, Lucrezia Togni ${ }^{1}$, Giovanni Giannatempo ${ }^{2}$, Vito Carlo Alberto Caponio ${ }^{2}$, \\ Marco Mascitti ${ }^{1}$ and Andrea Santarelli ${ }^{1}$ \\ 1 Department of Clinical Specialistic and Dental Sciences, Marche Polytechnic University, \\ 60126 Ancona, Italy; togni.lucrezia@gmail.com (L.T.); marcomascitti86@hotmail.it (M.M.); \\ andrea.santarelli@staff.univpm.it (A.S.) \\ 2 Department of Clinical and Experimental Medicine, University of Foggia, 71122 Foggia, Italy; \\ edottor@libero.it (G.G.); vito_caponio.541096@unifg.it (V.C.A.C.) \\ * Correspondence: eliluconi@gmail.com; Tel.: +39-071-220-6226 \\ + Presented at the XV National and III International Congress of the Italian Society of Oral Pathology and \\ Medicine (SIPMO), Bari, Italy, 17-19 October 2019.
}

Published: 10 December 2019

In the last years, the classification of odontogenic cysts and tumors has been highly debated, especially regarding odontogenic keratocyst (OKC). Indeed OKC, previously defined as keratocistic odontogenic tumor, has been re-introduced into the cyst group due to the lack of sufficient evidence to justify its classification as a neoplasm $[1,2]$. OKC is characterized by a thin keratinizing stratified squamous epithelial lining and a non-inflammatory fibrous connective wall. OKC may occur in association with the nevoid basal cell carcinoma syndrome (NBCCS), characterized by an autosomal dominant gene mutation with variable and usually high penetrance and expressivity.

Due to the almost restricted expression of p63 in epithelial cells, its role in the regulation of proliferation and differentiation of craniofacial-structure, this study investigated immunohistochemical expression of p63 both in solitary OKC and NBCCS-related OKC.

10 solitary OKC as well as 5 NBCCS-related OKC were analysed by immunohistochemistry for expression of p63. 4- $\mu \mathrm{m}$ serial sections were incubated with the monoclonal anti-p63 antibody (clone 4A4, Dako Cytomation, Glostrup, Denmark) diluted 1:50.

A semi-quantitative assessment of p63 expression was performed: "-" (stained cells: 0-5\%); "+" (stained cells: 6-30\%); "++" (stained cells: $31-50 \%) ; "+++"$ (stained cells: $>50 \%$ ). Staining intensity was graded on a scale of a semi-quantitative scale: 0 (no detectable); 1 + (weak); $2+$ (moderate); $3+$ (strong); $4+$ (very strong).

In NBCCS-related OKC 2 cases showed negligible p63 expression (0-5\%), 2 cases showed a percentage of stained cells of $6-30 \%$, and 1 case demonstrated a significant expression (31-50\%). In all solitary $\mathrm{OKC}$, the number stained cells were comprised between 0 and $5 \%$. Regarding staining intensity, in NBCCS-related OKC the nuclear staining of p63 was intense (3+) in all epithelial layers. On the contrary, in solitary OKC, the intensity of staining of p63 was moderate (2+) and reported only in basal-parabasal layer.

In conclusion, these data suggest that that p63 diffuse and intense immunostaining could be useful to distinguish solitary OKC from NBCCS-related OKC.

Conflicts of Interest: The authors declare no conflict of interest. 


\section{References}

1. El-Naggar, A.; Chan, J. WHO Classification of Head and Neck Tumours, 4th ed.; IARC: Lyon, France, 2017; pp. 235-237.

2. Lo Muzio, L.; Mascitti, M. Cystic lesions of the jaws: A retrospective clinicopathologic study of 2030 cases. Oral Surg. Oral Med. Oral Pathol. Oral Radiol. 2017, 124, 128-138, doi:10.1016/j.oooo.2017.04.006. 